\title{
Effects of Selenium Nanoparticles-loaded Chitosan Microspheres on Meat Selenium Content and Oxidative Stability in Broiler Chickens
}

\section{E Giamouri ${ }^{1}$, S Fortatos ${ }^{1}$, AC Pappas ${ }^{1}$, SN Yannopoulos ${ }^{2}$ and G Papadomichelakis ${ }^{1 *}$}

${ }^{1}$ Laboratory of Nutritional Physiology and Feeding, Department of Animal Science, School of Animal Biosciences, Agricultural University of Athens, Greece

${ }^{2}$ Foundation for Research and Technology Hellas - Institute of Chemical Engineering

Sciences (FORTH/ICE-HT), P.O. Box 1414, GR-26504, Rio-Patras, Greece

*Corresponding Author: G Papadomichelakis, Laboratory of Nutritional Physiology and Feeding, Department of Animal Science, School of Animal Biosciences,

Agricultural University of Athens, Greece.
Received: September 20, 2021

Published: October 20, 2021

(C) All rights are reserved by E Giamouri, et al.

\section{Abstract}

Dietary sodium selenite and selenium-yeast are commonly used to enhance the antioxidant defense in broilers, but they can be toxic. A new form of Se nanoparticles stabilized in chitosan microspheres (CS-SeNP) is well established for low toxicity, but their bioavailability and antioxidant potential has not been investigated in broiler feeding. Our objectives were: a) to synthesize and characterize the properties of CS-SeNP and b) to compare the effects of CS-SeNP as a dietary selenium source with those of sodium selenite and selenium-yeast on meat selenium concentration and oxidative stability in broiler chickens. The CS-SeNP were synthesized by chemical reduction and their properties were determined. Four experimental diets were offered to 200 broilers chickens $(5$ replicates/diet, 10 broilers/replicate); one control (C) with no added Se, and 3 diets supplemented with $0.4 \mathrm{mg}$ Se/kg either from sodium selenite + selenium-yeast (1:1 ratio; T1), selenium-yeast and CS-SeNPs (1:1 ratio; T2) or CS-SeNP alone (T3). Feed intake, weight gain and feed conversion ratio were monitored throughout the trial. At the end of the trial, 10 broilers per diet (2/replicate) were sacrificed and breast meat samples were collected. Meat fatty acid composition was determined by gas chromatography. Meat selenium content and oxidative stability were determined by hydride (vapor) generation atomic absorption spectroscopy and iron-induced lipid oxidation, respectively. Spherical monodispersed CS-SeNPs of $80.5 \pm 20 \mathrm{~nm}$ average diameter were obtained. The CS-SeNP were exclusively composed of elemental Se and were encapsulated in chitosan, as indicated by the X-ray diffraction and X-ray photoelectron spectroscopy surveys, respectively. Growth performance was not affected by the dietary selenium addition and differences were not observed between the dietary selenium sources. Meat selenium content and oxidative stability were similar in T1, T2 and T3 broilers, but significantly higher $(\mathrm{P}<0.05)$ when compared to $\mathrm{C}$ broilers. In conclusion, the selenium from CS-SeNP enriches meat with selenium and enhances meat oxidative stability in a manner similar to the commonly-used inorganic and organic forms. Given the well-established low toxicity, CS-SeNP has a very good potential as a dietary Se source in broilers and should be further studied. Keywords: Broilers; Oxidative Stability; Selenium; Selenium Nanoparticles-Loaded Chitosan Microspheres; Selenium-yeast; Sodium Selenite

\section{Abbreviations}

BSA: Bovine Serum Albumin; CS: Chitosan; DLS: Dynamic Light Scattering; CS-SeNP: Selenium Nanoparticles-Loaded Chitosan Mi- crospheres; DHA: Docosahexaenoic Acid (22:6n-3); FA: Fatty Acids; LC: Long-chain Fatty Acids; MDA: Malondialehyde; MUFA: Monoun- 
saturated Fatty Acids; PUFA: Polyunsaturated Fatty Acids; Se, Selenium; SeY: Se-Enriched Yeast; SS: Sodium Selenite; SeNP: Selenium Nanoparticles; SFA: Saturated Fatty Acids; XRD: X-ray Diffraction; XRS: X-ray Photoelectron Spectroscopy.

\section{Introduction}

Selenium (Se) is an integral part of at least 25 selenoproteins, which exert multiple biological activities regarding animal growth performance, fertility and disease prevention. Some of these selenoproteins are actively involved in antioxidant defense and redox regulation of the animals by controlling the levels of free radicals that are produced during normal metabolic activity [1]. Due to its antioxidant potential, dietary Se supplementation has been extensively investigated, among other antioxidants, as a key trace mineral for enhancing oxidative stability and shelf life of poultry meat, which is quite sensitive to oxidation owing to its high content of polyunsaturated fatty acids (PUFA) [2,3]. Studies in broilers have showed that feeding supplemental Se may significantly retard oxidation in liver and meat, in addition to tissue enrichment with Se [2-4].

The various forms of Se used in animal feeding have their pros and cons. The least expensive are inorganic Se salts, usually sodium selenite, but inorganic Se incorporation into tissues and antioxidant activity vary [6]. Organic Se, in the form of selenomethionine is more efficiently incorporated into tissues and it is more bioactive, but its production process makes it more expensive [1]. Both forms can be toxic when provided at excessive levels i.e. well above the nutritional requirements. In fact, the margin between beneficial and toxic effects of Se is considered narrow [5-7]. To ensure feed safety, maximum levels for Se in complete feeds have been set at $0.5 \mathrm{mg} / \mathrm{kg}$ in the European Union [8] and $0.3 \mathrm{mg} / \mathrm{kg}$ in the United States [9]. However, there is increasing concern in the animal industry that these recommended levels may not be adequate to supply animals with the Se needed to cope with the stressful commercial rearing conditions; therefore, research has turned to alternative Se forms with potentially higher bioavailability and/or lower toxicity [10].

The recent advancements in nanotechnology brought into light the use of nanoparticles, which are widely envisaged due to their innovative features such as a high surface activity, high catalytic efficiency, large surface area, strong adsorbing ability, but most im- portantly low toxicity [11-13]. Selenium nanoparticles (SeNP) in particular, are inorganic elemental selenium nano-particles, which can retain the biological activity, increase Se availability and reduce the toxicity of the Se element [14]. They can be very efficient and low-toxicity nutritional additives [15]. It has been reported that SeNP possess comparable efficiency to selenite and methyl selenocysteine in upregulating selenoenzymes but with dramatically decreased toxicity $[16,17]$. Indeed, SeNP can improve the growth performance and the antioxidant status, as well as meat quality indices and Se concentration, in broilers [18-20]. The synthesis of SeNP can be easily achieved by biological reduction in the oxyanions, selenite and selenite [21], or by chemical means [22-24]. However, SeNPs usually enlarge, aggregate and finally transform into a gray/black analog that is biologically inert [25]. To address this problem, several capping agents (also called stabilizers) have been utilized to preserve SeNP physically separated, among which chitosan (CS) has gained considerable attention [25]. The CS is a positively charged natural polysaccharide polymer with excellent biodegradable and biocompatible characteristics [22]. On the other hand, it may retard the release of SeNP due to the deficiency of the enzymes to deal with CS in some species of animals and human beings [22]. Hence, it is not clear how effectively the CS-SeNP can be solubilized in the gastrointestinal tract of animals, and permit the release, absorption, and incorporation of Se into tissues [1]. Recent evidence indicates that SeNP stabilization using chitosan seems to be a viable alternative and presents great application potential in aquaculture, because they exert effects on growth performance, feed efficiency, and antioxidative capacity of fish [7]. To our knowledge, the potential of CS-SeNP as a dietary Se source has not been investigated until today in broilers; only BSA (bovine serum albumin) stabilized SeNP have been tested. Therefore, the present study sought to a) synthesize and characterize the physico-chemical properties of CS-SeNP and b) compare the effects of CS-SeNP on meat Se content and oxidative stability with those of sodium selenite (SS) and Se-yeast (SY) in broiler chickens, at the recommended by the EU dietary levels.

\section{Materials and Methods}

Synthesis and characterization of selenium nanoparticlesloaded chitosan microspheres

Selenium nanoparticles-loaded chitosan microspheres (CSSeNP) were synthesized by reducing sodium selenite in the presence of chitosan, which adheres to Se atoms and controls the size 
of their aggregation according to the method of Bai., et al. [22] with some modifications. Briefly, $1.0 \mathrm{~g}$ of chitosan (CS, molecular weight 50-190 kDa, 90\% deacylated chitin; Sigma-Aldrich) and $1.6 \mathrm{~g}$ of ascorbic acid were dissolved in $100 \mathrm{ml}$ of $1 \%(\mathrm{w} / \mathrm{w})$ acetic acid. Subsequently, $10 \mathrm{ml}$ of an aqueous solution containing $0.4 \mathrm{~g}$ of sodium selenite was added dropwise into the CS/ascorbic acid solution and stirred continuously (600-800 rpm) to obtain a red SeNP-M colloid. The colloid was dialyzed against $1 \%(\mathrm{w} / \mathrm{w})$ acetic acid (3.5 kDa MWCO dialysis bags) for 6 hours to remove the excess ascorbic acid and other by-products. After that, the colloid was well mixed with a clean CS solution (3 g CS in $100 \mathrm{ml}$ of aqueous $1 \%$ acetic acid) to achieve final concentrations of $0.09 \%(w / w)$ Se and $2.0 \%(\mathrm{w} / \mathrm{w}) \mathrm{CS}$. The final colloid was lyophilized and the cake was milled through a 0.5-mm sieve (Cyclotec, 1093 sample mill; Tecator, Höganäs, Sweden) to obtain a bright red free flowing powder, which was stored at room temperature until use.

The hydrodynamic sizes of the CS-SeNP were measured using dynamic light scattering (DLS) on Mastersizer particle size and zeta potential analyzer (Malvern Instruments, Malvern, UK). The X-ray diffraction (XRD) was used to study the structure of the CS-SeNP and was performed in the range $5^{\circ} \leq 2 \theta \leq 90^{\circ}$ with the aid of a D8 Advance diffractometer (Bruker Co, Billerica, MA, US), which utilizes the $\mathrm{CuKa}(1.5406 \AA$ ) radiation source operated at $40 \mathrm{kV}$ and 40 $\mathrm{mA}$. The X-ray photoelectron spectroscopy (XPS) was used to study the composition of the CS-SeNP and the experiments were carried out in an ultra-high vacuum system using the unmonochromatized AlK $\alpha$ line (1253.6 eV). Two analyzer pass energies were used, one at $20 \mathrm{eV}$ (resulting in full width at half maximum of $0.9 \mathrm{eV}$ for the Ag3d5/2 peak) and another at $40 \mathrm{eV}$. The XPS core-level spectra were analyzed using a fitting routine, which can decompose each spectrum into individual mixed Gaussian-Lorentzian peaks after a Shirley background subtraction. Errors in our quantitative data were found in the range of $\sim 10 \%$, (peak areas) while the accuracy for binding energies (BE) was approximately $0.1 \mathrm{eV}$. The analyzed area on the sample surface was a rectangle with dimensions $7.0 \times$ $15 \mathrm{~mm}^{2}$.

\section{Animals, diets and experimental procedures}

A total of 200 as hatched Ross broiler chickens were used. The broilers were obtained from a commercial hatchery. All chickens had similar Se status because the broiler breeder flock was fed a commercial diet with $0.20 \mathrm{mg}$ Se per $\mathrm{kg}$ of diet. There were 5 rep- licate pens of 4 dietary treatments namely C, T1, T2 and T3 and T4 randomly allocated in the house. There were 10 chickens per pen, 50 per treatment. The broilers of a control group (C) were fed a basal diet (BD), without any Se supplementation; the only Se present was that of feed ingredients (Table 1). The T1 broilers were fed the basal diet supplemented with $0.2 \mathrm{mg} \mathrm{Se} / \mathrm{kg}$ from sodium selenite (SS) and $0.2 \mathrm{mg} \mathrm{Se} / \mathrm{kg}$ from a yeast source (SeY; Sel-Plex ${ }^{\circledR}$, Alltech Inc, Nicholasville, KY, US). The T2 broilers were fed the basal diet supplemented with $0.2 \mathrm{mg} \mathrm{Se} / \mathrm{kg}$ from SeY and $0.2 \mathrm{mg} \mathrm{Se} / \mathrm{kg}$ from CS-SeNP, which were synthesized in our laboratory as described above. Finally, the T3 broilers were fed the basal diet supplemented with $0.4 \mathrm{mg} \mathrm{Se} / \mathrm{kg}$ from CS-SeNP only. Limestone was used as a carrier to dilute the concentration of CS-SeNP to a suitable level for blending the ration to achieve the desired levels for diet formulation. The ingredient composition of the diets is shown in table 1.

The duration of the trial was 6 weeks with housing and care of broilers conforming to the guidelines of the Department of Animal Science and Aquaculture of the Agricultural University of Athens. The experimental protocol was approved by the AUA Bioethics Committee (no. 13/16-03-2021). The broilers were raised, according to Ross's management manual, in a house where light and ventilation were controlled. The broilers were fed a starter diet to the $10^{\text {th }}$ day of their life, a grower diet to the $24^{\text {th }}$ day and a finisher diet thereafter (until 42 days of age). Feed and water were provided ad libitum. Each growing phase diet (starter, grower and finisher) contained the same level of added Se which was appropriate for each experimental treatment (Table 1). The lighting program was $23 \mathrm{~h}$ of light and $1 \mathrm{~h}$ of dark. Stocking density was according to EU legislation. At the end of each growth phase (day 10, 24 and 42), broilers were weighted and the body weight was recorded. Furthermore, feed intake was measured weekly and the feed to gain ratio was calculated. At the end of the experiment (42 days of age), two broilers per pen were sacrificed by electro stunning and exsanguination, eviscerated and after a $24 \mathrm{~h}$ chilling period at $4^{\circ} \mathrm{C}$, carcasses were weighed and dressing percentage was determined. Subsequently, breast samples from the Pectoralis major muscle were collected. In detail, from each carcass, the right part of the breast was excised, vacuum packed and stored at $-20^{\circ} \mathrm{C}$ until analyzed for FA. A smaller portion (ca. 2-3 g) from the right part of the breast was used for the determination of Se concentration. Simultaneously, the left part of the breast was removed, vacuum packed and stored at $-20^{\circ} \mathrm{C}$, to determine lipid oxidation. 


\begin{tabular}{|c|c|c|c|c|}
\hline Ingredients & Starter & $\begin{array}{l}\text { Grow- } \\
\text { er }\end{array}$ & \multicolumn{2}{|c|}{ Finisher } \\
\hline Maize & 48.47 & 52.09 & \multicolumn{2}{|r|}{57.60} \\
\hline Soybean meal & 42.84 & 38.98 & \multicolumn{2}{|r|}{33.39} \\
\hline $\begin{array}{l}\text { Vitamin and } \\
\text { Mineral Premix }{ }^{1}\end{array}$ & 0.20 & 0.20 & \multicolumn{2}{|r|}{0.20} \\
\hline Limestone & 1.41 & 1.28 & \multicolumn{2}{|r|}{1.16} \\
\hline $\mathrm{NaCl}$ & 0.40 & 0.40 & \multicolumn{2}{|r|}{0.40} \\
\hline Methionine & 0.36 & 0.31 & \multicolumn{2}{|r|}{0.28} \\
\hline Soybean oil & 4.47 & 5.18 & \multicolumn{2}{|r|}{5.61} \\
\hline Lysine & 0.24 & 0.17 & \multicolumn{2}{|r|}{0.18} \\
\hline Threonine & 0.10 & 0.07 & \multicolumn{2}{|r|}{0.04} \\
\hline $\begin{array}{l}\text { Monocalcium } \\
\text { Phosphate }\end{array}$ & 1.43 & 1.24 & \multicolumn{2}{|r|}{1.06} \\
\hline Choline & 0.08 & 0.07 & \multicolumn{2}{|r|}{0.08} \\
\hline & & & \multicolumn{2}{|c|}{ Se level (mg/kg) } \\
\hline \multicolumn{3}{|l|}{ Diet } & $\begin{array}{l}\text { Add- } \\
\text { ed }^{2}\end{array}$ & Determined $^{3}$ \\
\hline $\mathrm{C}$ & \multirow{4}{*}{$\begin{array}{l}\text { Se not } \\
\text { Deter- } \\
\text { mined }\end{array}$} & \multirow{4}{*}{$\begin{array}{l}\text { Se not } \\
\text { Deter- } \\
\text { mined }\end{array}$} & - & $0.117 \pm 0.020$ \\
\hline $\mathrm{T} 1$ & & & 0.40 & $0.492 \pm 0.049$ \\
\hline $\mathrm{T} 2$ & & & 0.40 & $0.504 \pm 0.056$ \\
\hline T3 & & & 0.40 & $0.488 \pm 0.045$ \\
\hline
\end{tabular}

\section{Determination of selenium in CS-SeNP, diets and meat}

Selenium concentration in feed, CS-SeNP and breast samples was determined using an Agilent 240FS AA atomic absorption spectrometer fitted with VGA77 Vapor generation accessory (Agilent, Santa Clara, USA) based on the method described by Pappas., et al. [26] with minor modifications. In brief, samples ( $0.50 \mathrm{~g}$ ) were soaked in $4 \mathrm{ml}$ of concentrated $\mathrm{HNO}_{3}(65 \% \mathrm{w} / \mathrm{v}$, Suprapur; Merck, Germany). The samples were left for 30 minutes for pre-digestion. Then, another $6 \mathrm{ml}$ of concentrated $\mathrm{HNO}_{3}$ were added and then were heated in a microwave accelerated digestion system (CEM, Mars X-Press, Matthews, NC, USA) according to the following program: power was ramped during $20 \mathrm{~min}$ from 100 to $1200 \mathrm{~W}$ and held for $15 \mathrm{~min}$. The temperature reached a maximum of $200^{\circ} \mathrm{C}$ followed by a cool-down cycle for $15 \mathrm{~min}$. Losses of volatile element compounds did not occur as the tubes were sealed during heating. The samples were then filtered with disposable syringe filters $0.20 \mu \mathrm{m} / 15 \mathrm{~mm}$ (Chromafil, Macherey-Nagel, Germany). Se $\mathrm{VI}^{\mathrm{VI}}$ was reduced to $\mathrm{Se}^{\mathrm{IV}}$ by warming with $6 \mathrm{M}$ concentrated HCL (Merck, Germany) before analysis in the AA atomic absorption system. Calibration standard solutions were prepared from high purity standards. Reductant agent $\mathrm{NaBH}_{4} \quad 0.6 \%$ w/v (Sigma- Aldrich, USA) and $\mathrm{NaOH}$ 0.5\% w/v (Fisher Scientific, UK) and $10 \mathrm{M} \mathrm{HCl}$ (Merck, Germany) was used for vapor generation. To assess the accuracy of the process, two standard reference materials were used, namely RM8414 (Bovine muscle powder, National Research Council, Canada) and RM1577c (Bovine liver, National Institute of Standards and Technology, USA).

\section{Determination of fatty acid profile}

The FA of diets and intramuscular fat were extracted and methylated directly [27]. Any external fat and connective tissue were dissected out of the breast samples, which were then blended in a domestic food processor until smooth. Duplicate $1( \pm 0.05)$ g samples were hydrolyzed for $1.5 \mathrm{~h}$ at $55^{\circ} \mathrm{C}$ in $1 \mathrm{~N}$ potassium hydroxide in methanol, containing a known amount (approximately $0.5 \mathrm{mg}$ ) of tridecanoic acid (C13:0) as internal standard. The potassium hydroxide was then neutralized, and the free FAs were methylated by sulphuric acid catalysis $\left(24 \mathrm{~N} \mathrm{H}_{2} \mathrm{SO}_{4}\right)$ for $1.5 \mathrm{~h}$ at $55^{\circ} \mathrm{C}$. Hexane ( 3 $\mathrm{ml}$ ) was added to the reaction tube, which was vortex-mixed and centrifuged at $1100 \mathrm{~g}$. The supernatant hexane layer containing the FA methyl esters (FAME) was kept at $-20^{\circ} \mathrm{C}$, until analyzed by gas chromatography. A temperature-programmed run was followed on a Perkin Elmer Autosystem XL gas chromatograph equipped with

${ }^{3}$ Average of 4 samples per diet \pm standard deviation.

Citation: E Giamouri, et al. "Effects of Selenium Nanoparticles-loaded Chitosan Microspheres on Meat Selenium Content and Oxidative Stability in Broiler Chickens". Acta Scientific Veterinary Sciences 3.11 (2021): 27-38. 
a $30 \mathrm{~m} \times 0.25 \mathrm{~mm} \times 0.25 \mu \mathrm{m}$ internal diameter HP-Innowax capillary column (Agilent Technologies, J\&W GC columns, Santa Clara, CA, USA) and a flame ionization detector (FID). The column temperature was programmed for $1 \mathrm{~min}$ at $140^{\circ} \mathrm{C}$, raised by $2.50 \mathrm{C} / \mathrm{min}$ to $200^{\circ} \mathrm{C}$, then to $230^{\circ} \mathrm{C}$ by $10 \mathrm{C} / \mathrm{min}$ and held for $1 \mathrm{~min}$, and finally to $240^{\circ} \mathrm{C}$ by $4^{\circ} \mathrm{C} / \mathrm{min}$ and held for $10 \mathrm{~min}$. Helium was the carrier gas at a constant pressure of 18 psi and the temperature of both the injector and FID was set at $250^{\circ} \mathrm{C}$. Fatty acids were identified by comparison with standards purchased from Sigma-Aldrich Co. (FAME 37 Component; Sigma-Aldrich Co. Supelco, IL, USA) and quantification was achieved using the internal standard (13:0) added prior to hydrolysis. Total weights of FA (mg/100g) in diets were calculated as the sum of areas for all FA peaks compared to the area for $0.5 \mathrm{mg}$ internal standard. The FA were expressed as \% by weight of total FA.

\section{Determination of iron-induced lipid oxidation}

The malondialdehyde (MDA) formed during iron-induced lipid oxidation with a Fenton reaction mixture [28,29] was assessed. Muscle samples of $2 \mathrm{~g}$ were homogenized in a X 1000D model homogenizer (CAT, M. Zipperer GmbH, Germany) with $20 \mathrm{ml}$ of 0.15 $\mathrm{M} \mathrm{KCl} \mathrm{(pH} \mathrm{7.2)} \mathrm{for} 1 \mathrm{~min}$ at 12,000 rpm in an ice bath. The homogenate was centrifuged at $2000 \mathrm{~g}$ for $10 \mathrm{~min}$ at $4^{\circ} \mathrm{C}$ and $0.5 \mathrm{ml}$ of the supernatant was mixed with $0.5 \mathrm{ml}$ of $0.15 \mathrm{M} \mathrm{KCl}, 30 \mu \mathrm{l}$ of 3 $\mathrm{mM}$ BHT (time point 0 samples). Five milliliters of the supernatant were incubated at $37^{\circ} \mathrm{C}$ in a water bath, under agitation, with $5 \mathrm{ml}$ of $0.5 \mathrm{mM} \mathrm{FeSO}_{4}$ and $1 \mathrm{mM} \mathrm{H}_{2} \mathrm{O}_{2}$ solution $(50 \mu \mathrm{l})$ for 30,120 and 300 min. After each incubation time, $1 \mathrm{ml}$ was taken and the oxidation reaction stopped by the addition of $30 \mu \mathrm{l}$ of $3 \mathrm{mM}$ BHT (time points 30, 120 and 300 samples). The procedure for MDA measurement for all the time points samples was carried out as follows: $1 \mathrm{ml}$ of TBA-TCA solution (35 mM TBA and 10\% TCA in $125 \mathrm{mM} \mathrm{HCl}$ ) was added and samples were placed in a boiling water bath for $30 \mathrm{~min}$. After cooling in an ice bath for 5 min and standing at room temperature for $45 \mathrm{~min}$, the pink chromogen was extracted with $4 \mathrm{ml}$ of n-butanol and phase separation by centrifugation at $3000 \mathrm{~g}$ for $10 \mathrm{~min}$. The absorbance of the supernatant was measured at 535 $\mathrm{nm}$ (Helios $\alpha$, Thermo spectronic, Cambridge, UK) immediately after transferring the n-butanol into the cuvettes because the pink chromogen was stable for a maximum of 30 min only. The concentration of MDA was calculated using its molar extinction coefficient $\left(156,000 \mathrm{M}^{-1} \mathrm{~cm}^{-1}\right)$. Results were expressed as mg MDA per $\mathrm{kg}$ of wet meat.

\section{Statistical analysis}

Data were analyzed using the SPSS statistical package (version 17.0) and are presented as means \pm standard error (SEM). Feed intake, body weight gain, feed conversion ratio, cold carcass weight, dressing percentage and Se content, FA profile in the LL muscle were analyzed by one-way analysis of variance (ANOVA) with diet as fixed effect and post-hoc tests were carried out. The MDA values were analyzed using a repeated measures ANOVA with diet and time as fixed effects, and post-hoc tests were carried out within each time point. Post-hoc tests were conducted based on Tukey's criterion. Statistical significance was set at $\mathrm{P}<0.05$ for all tests.

\section{Results and Discussion}

Characterization of the Se nanoparticles-loaded chitosan microspheres

The DLS analysis showed that monodispersed spherical CSSeNP with an average diameter of $80.5 \pm 20 \mathrm{~nm}$ (mean \pm s.d.) were produced. The XRD pattern showed an amorphous structure of the Se in the CS-SeNP, since no traces of Bragg reflections related to crystalline Se were observed (Fig. 1). The XPS survey scans revealed the presence of $\mathrm{C}, \mathrm{O}$, and $\mathrm{N}$ atoms on the sample surface (Fig. 2a). The C1s peak was deconvoluted into three components as shown in Fig. 2b. The band located at $284.7 \mathrm{eV}$, could be assigned to $\mathrm{C}-\mathrm{C}$ or adventitious carbon, which confirmed the presence of the aliphatic group. The band at $286.3 \mathrm{eV}$ was associated with $\mathrm{C}-\mathrm{N}$ and $\mathrm{C}-\mathrm{O}$ groups, and the band at $288 \mathrm{eV}$ was attributed to $\mathrm{C}=\mathrm{O}$ or $\mathrm{O}-\mathrm{C}-\mathrm{O}$ species. In addition, the N1s peak centered at $399.3 \mathrm{eV}$ (Fig. 2c), could be assigned to $-\mathrm{NH}_{2}$ and - $\mathrm{NH}$ groups. The Se3d peak centered at $55.3 \mathrm{eV}$ (Fig. 2d) confirming that the valence state of Se in CSSeNP was zero $\left(\mathrm{Se}^{0}\right)$. XPS. High concentrations of C, $\mathrm{O}$, and $\mathrm{N}$ atoms (67.97, 28.67 and $3.12 \%$, respectively) corresponding to CS along with traces of Se $(0.25 \%)$ were found on the surface of SeNP-M. The atomic absorption analysis showed a content of $20.000 \mathrm{mg} \mathrm{Se} /$ kg CS-SeNP.

The CS-SeNP are not commercially available. They are synthesized in laboratory and the characterization of their physico-chemical properties is a common practice prior to experimental use. This is because the conditions during preparation largely affect their characteristics [24]. Therefore, our first concern was to confirm that the synthesis adopted in our laboratory had the desired results regarding size, encapsulation in CS, and nature and crys-

Citation: E Giamouri, et al. "Effects of Selenium Nanoparticles-loaded Chitosan Microspheres on Meat Selenium Content and Oxidative Stability in Broiler Chickens". Acta Scientific Veterinary Sciences 3.11 (2021): 27-38. 


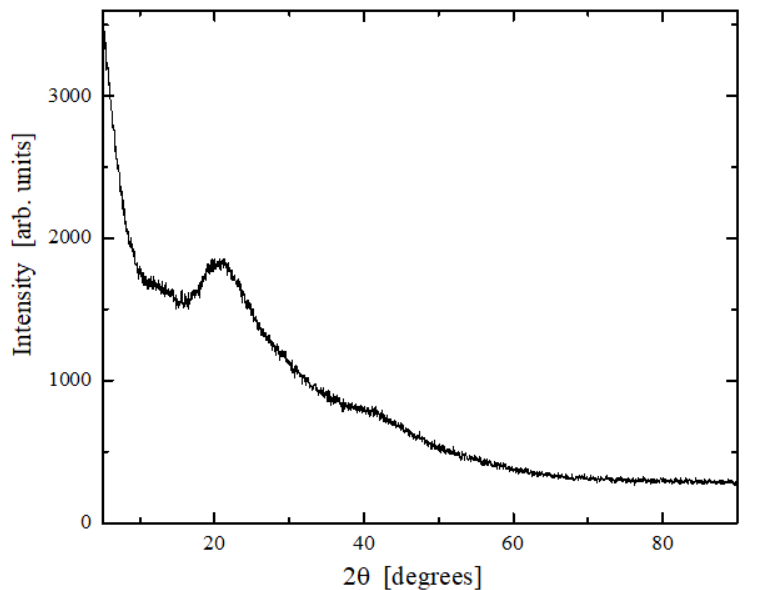

Figure 1: X-ray diffraction (XRD) patterns of the selenium nanoparticles-loaded chitosan microspheres (CS-SeNP-M).
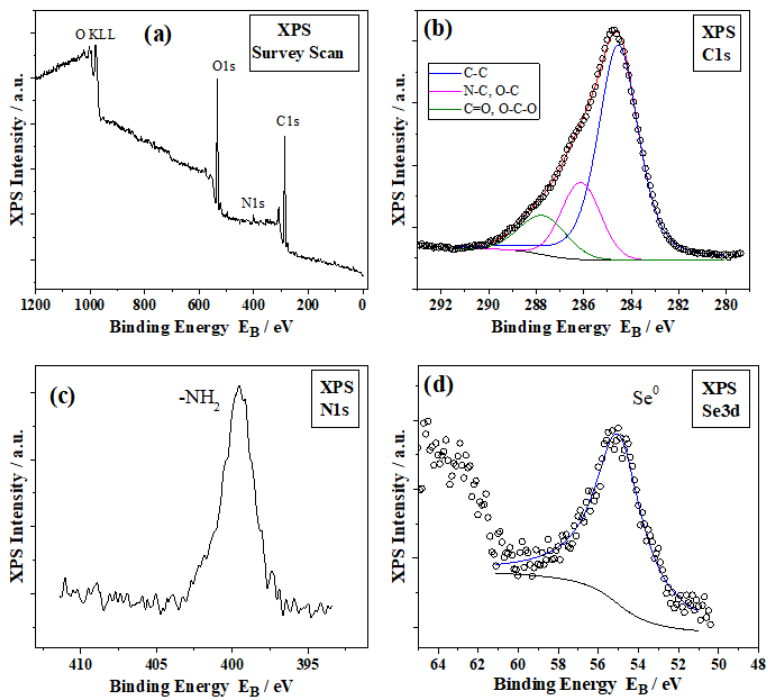

Figure 2: (a) Survey X-ray photoelectron spectroscopy (XPS) spectrum of the selenium nanoparticles-loaded chitosan microspheres (SeNP-M). (b) Deconvolution of the C1s XPS spectrum into three components. (c) N1s XPS spectrum. (d)

Se3d XPS spectrum. Experimental data appear as open circles in (b) and (d), while solid lines are fitting results. tallinity of Se. The size distribution analysis was conducted in the final colloid before lyophilization. The results showed an acceptable size $(80.50 \pm 20.0 \mathrm{~nm})$ very close to that reported in previous studies using CS [1,7,22,25,30-32] or other capping agents [33,34]. Small size $(<200 \mathrm{~nm})$ is critical because it favors the absorption and the biological activity of SeNP [5].

Traces of Se were detected during the XPS survey in the present work. Since XPS measures the characteristics of the CS-SeNP'S surface, it is concluded that all of the Se was successfully embedded inside the CS shell in aggrement with earlier studies [22,25,32]. Therefore, the synthesized CS-SeNP were expected to have a good stability (would not easily aggregate into large particles) thereby maintaining their biological activity in the gastrointestinal tract of the animals. The elemental nature of Se in the CS-SeNP was also confirmed by both the XRD and XPS surveys, in agreement with earlier works $[7,22,24,25]$. The elemental Se in the CS-SeNP prepared herein was amorphous, as also reported by Filipović., et al. [24] and other researchers [33,34]. This amorphous structure however, was in contrast to the crystalline (trigonal phase) feature reported in the method we adopted for the synthesis of CS-SeNP [22], and in other works [25,32]. It is not unlikely that differences in the synthesis conditions of CS-SeNP between studies may have affected the crystallinity of Se. Zhang., et al. [32] observed that amorphous elemental Se was present in CS-SeNP encapsulated in high molecular weight CS (200 kDa), whereas crystalline elemental Se was obtained with low molecular weight CS (3 kDa). The crystalline nature of Se when using a very low molecular weight CS (3 $\mathrm{kDa}$ ) was also reported by Bai., et al. $[22,25]$. It has been also found that the use of high temperatures $\left(90^{\circ} \mathrm{C}\right)$ to dissolve CS during the synthesis of CS-SeNP results in a crystallic structure of Se, which negatively affects its biological activity [24]. Herein, we used a moderate to high molecular weight CS (50-190 kDa) and mild temperatures $\left(<50^{\circ} \mathrm{C}\right)$ during synthesis, which explain the amorphous structure of Se. In general, CS-SeNP with desired properties (smallsized with amorphous elemental Se) were easily prepared in the laboratory and produced in large quantities for the needs of the experiment quite fast. This indicates that CS-SeNP synthesis could be up-scaled to industrial production as the method adopted here was economic and environment-friendly [22].

\section{Growth performance and carcass traits}

Feed intake, weight gain, feed conversion ratio and carcass 


\begin{tabular}{|l|c|c|c|}
\hline Element & $\begin{array}{c}\text { Binding } \\
\text { energy }(\mathbf{e V})\end{array}$ & $\begin{array}{c}\text { Concentration } \\
\mathbf{( \% )}\end{array}$ & Assignment \\
\hline 01s & 532.53 & $28.67 \pm 0.05$ & C-O \\
\hline C1s & 284.75 & $67.97 \pm 0.06$ & C-C, C-N, O-C \\
\hline N1s & 399.55 & $3.12 \pm 0.04$ & $-\mathrm{NH}_{2},-\mathrm{NH}$ \\
\hline Se3d & 55.28 & $0.25 \pm 0.01$ & Se $(0)$ \\
\hline
\end{tabular}

Table 2: Percent relative atomic concentration of elements on the surface of the selenium nanoparticles-loaded chitosan microspheres (CS-SeNP) as determined using the peak areas of the X-ray photoelectron spectroscopy (XPS) spectra.

traits were not affected by SS, SeY or CS-SeNP supplemented diets in comparison with the unsupplemented $\mathrm{C}$ diet, for the whole experimental period (Table 3 ).

Cai., et al. [10] also did not observe any positive response of broilers to dietary SeNP supplementation, throughout a 42-day

\begin{tabular}{|l|c|c|c|c|c|c|}
\hline \multirow{2}{*}{} & \multicolumn{4}{|c|}{ Diet $^{\mathbf{1}}$} & \multirow{2}{*}{ SEM $^{2}$} & \multirow{2}{*}{ P-value } \\
\cline { 2 - 6 } & C & T1 & T2 & T3 & & \\
\hline Initial BW, g & 46.9 & 46.8 & 46.9 & 46.8 & 0.46 & 0.989 \\
\hline Final BW, g & 3355.6 & 3299.4 & 3246.0 & 3144.7 & 134.70 & 0.555 \\
\hline ADFI, g/d & 125.8 & 123.2 & 123.6 & 119.5 & 3.82 & 0.542 \\
\hline ADWG, g/d & 78.8 & 77.4 & 76.2 & 73.8 & 3.21 & 0.554 \\
\hline FCR, g/g & 1.60 & 1.59 & 1.63 & 1.62 & 0.032 & 0.672 \\
\hline $\begin{array}{l}\text { Cold carcass } \\
\text { weight, g }\end{array}$ & 2558.0 & 2674.0 & 2471.5 & 2537.5 & 79.37 & 0.121 \\
\hline $\begin{array}{l}\text { Dressing } \\
\text { percentage, \% }\end{array}$ & 76.3 & 77.0 & 76.5 & 75.7 & 0.66 & 0.308 \\
\hline
\end{tabular}

Table 3: Effects of diet on body weight (BW), average daily feed intake (ADFI), average daily weight gain (ADWG), feed conversion ratio (FCR) $(n=50$ broilers/diet) and slaughter traits $(n=50$ broilers/diet).

${ }^{1}$ Se source: a) sodium selenite $(0.2 \mathrm{mg} / \mathrm{kg})$ and Se-yeast $(0.2 \mathrm{mg} /$ $\mathrm{kg}$ ) in T1 diet, b) Se-yeast (0.2 mg/kg) and CS-SeNP (selenium nanoparticles-loaded chitosan microspheres, $0.2 \mathrm{mg} / \mathrm{kg}$ ) in T2 diet and c) CS-SeNP (0.4 mg/kg) in T3 diet; Se-yeast was in the form of Sel-Plex ${ }^{\circledR}$ (Alltech Inc., Nicholasville, KY, USA); no Se was added to the control (C) diet.

${ }^{2} \mathrm{SEM}=$ Standard error of means.

experimental duration. On the other hand, Zhou and Wang [18] reported that SeNP supplementation of chicken diets with $0.30 \mathrm{mg} /$ $\mathrm{kg}$ was effective in increasing the growth performance and feed conversion ratio of the animals. In the latter study, it was also underlined that the basal diet used resulted in a Se deficiency because in most areas of China, the natural Se content of feedstuffs used in poultry feeding is very low [18]; hence, the increased growth performance was the result of meeting the nutritional requirements by supplementing Se in the diets and not the source of Se per se. Other studies also showed that dietary Se addition improves live weight in Se-depleted broilers at hatch [35]. It must be noted that most studies of dietary Se supplementation in broilers do not discover any effect of Se on growth performance and feed conversion ratio [10]. It appears that the efficacy of Se (either from inorganic and organic or nanoparticle sources) is exerted when the basal diet is Se-deficient or under commercial production conditions, where broilers may encounter several challenges (heat stress, microbes, mycotoxins and severe oxidative stress) [10]. Interestingly, the same situation has been observed in other farm animals like rabbits, where it was reported that Se affects growth performance only when the basal diet is Se-deficient or when farm conditions are harsh [36-38].

Meat selenium content, oxidative stability and fatty acid composition

The T1, T2 and T3 diets supplemented with Se increased significantly $(\mathrm{P}<0.05$ ) Se content in breast meat of chicken by 164,184 and $169 \%$, respectively, in comparison with the control diet. No differences in the Se content in breast meat were found between T1, T2 and T3 diets (Table 4).

The present results revealed that the Se content in breast meat of chickens was significantly increased by supplementing the diets with CS-SeNP. Similar effects have been observed in previous studies using Se nanoparticles (SeNP). Zhou and Wang [18] reported an increase in muscle Se content with dietary SeNP supplementation. In addition, Hu., et al. [39] reported that Se from SeNP was more efficiently retained in the body than SS. In the present study, such a conclusion could be based only on a comparison between $\mathrm{T} 1(\mathrm{SS}+\mathrm{SeY})$ and $\mathrm{T} 2$ diets (SeY+CS-SeNP) which eliminates the residual effect of SeY. It seems that Se absorption from CS-SeNP tends to be numerically higher compared to SS, but the difference was not significant. A notable difference between our study and that of Hu., et al. [39] is the stabilizer used during SeNP synthesis. Hu., 


\begin{tabular}{|c|c|c|c|c|c|c|}
\hline & \multicolumn{4}{|c|}{ Diet $^{1}$} & \multirow{2}{*}{ SEM $^{2}$} & \multirow{2}{*}{$P$-value } \\
\hline & C & T1 & $\mathrm{T} 2$ & T3 & & \\
\hline $\begin{array}{l}\text { Se content, } \\
\mathrm{mg} / \mathrm{kg}\end{array}$ & $0.121^{\mathrm{a}}$ & $0.199^{b}$ & $0.223^{b}$ & $0.204^{\mathrm{b}}$ & 0.0268 & 0.008 \\
\hline \multicolumn{7}{|l|}{ MDA, mg/kg } \\
\hline $0 \mathrm{~min}$ & 0.176 & 0.150 & 0.150 & 0.160 & 0.0235 & 0.656 \\
\hline $30 \mathrm{~min}$ & 0.317 & 0.318 & 0.277 & 0.241 & 0.0347 & 0.137 \\
\hline $120 \mathrm{~min}$ & $0.528^{a}$ & $0.434^{\mathrm{ab}}$ & $0.329^{\mathrm{b}}$ & $0.372^{\mathrm{b}}$ & 0.0683 & 0.009 \\
\hline $300 \mathrm{~min}$ & $0.819^{\mathrm{a}}$ & $0.644^{\mathrm{ab}}$ & $0.477^{\mathrm{b}}$ & $0.471^{\mathrm{b}}$ & 0.0878 & 0.015 \\
\hline
\end{tabular}

radicals. Free radicals are normally produced by the gastrointestinal cells during digestion and absorption of nutrients, and have the ability to cleave the1,4- $\beta$-d-glucoside bonds of CS leading to its depolymerization $[32,41]$. This cleavage is more pronounced in moderate to high molecular weight CS because of the incompact structure of the shell that covers the SeNP [32]. Thus, it is evident from the literature and the present results that CS is soluble in the gastrointestinal tract and that Se is adequately released for absorption and incorporation into tissues.

Table 4: Effects of diet on breast selenium (Se) content and malondialdehyde (MDA) concentrations during iron-induced lipid oxidation ( $\mathrm{n}=10$ broilers/diet).

Different superscripts denote significant difference $(\mathrm{P}<0.05)$.

${ }^{1}$ Se source: a) sodium selenite $(0.2 \mathrm{mg} / \mathrm{kg})$ and Se-yeast $(0.2 \mathrm{mg} /$ $\mathrm{kg}$ ) in T1 diet, b) Se-yeast $(0.2 \mathrm{mg} / \mathrm{kg}$ ) and CS-SeNP (selenium nanoparticles-loaded chitosan microspheres, $0.2 \mathrm{mg} / \mathrm{kg}$ ) in T2 diet and c) CS-SeNP $(0.4 \mathrm{mg} / \mathrm{kg})$ in T3 diet; Se-yeast was in the form of Sel-Plex ${ }^{\circledR}$ (Alltech Inc., Nicholasville, KY, USA); no Se was added to the control (C) diet.

${ }^{2}$ SEM $=$ Standard error of means.

et al. used BSA, whereas CS was used herein. Generally, BSA as a hydro-soluble protein could be easily dissolved in the digestive tract of animals thereby releasing SeNP faster. In contrast, CS is a carbohydrate polymer, which might retard the release of SeNP due to the deficiency of the enzymes to deal with it in some species of animals and humans [22]. Nevertheless, the bioavailability of the CS-SeNP was acceptable and comparable to that of SS and SeY, as indicated by the Se content in breast meat herein. It appears that despite its enzyme-resistant nature, CS was dissolved in the gastrointestinal tract of the broilers and Se was released, absorbed and incorporated into breast meat. Similar results have been reported in fish studies [7]. The release of Se from a polymeric matrix like CS is a function of many variables, including diffusion through the polymer matrix, polymer erosion, Se affinity to the polymer, and swelling degree of the polymer $[7,40]$. CS is an acid-soluble polymer, which may be eroded by the stomach acids to some extent and subsequently swell in the intestine environment, as has been verified by in vitro simulations in gastric and intestinal fluids [7,22,32]. Another possible explanation is the susceptibility of CS to free

Our study showed that the MDA in T1, T2 and T3 fed broilers showed significantly $(\mathrm{P}<0.05)$ lower concentrations compared to the $\mathrm{C}$ ones after 300 min of oxidation (Table 4). A trend for lower MDA concentration was observed in T2 and T3 fed broilers when compared to T1 after $300 \mathrm{~min}$ of oxidation. These results indicated the protective role of Se (from SS, SeY or CS-SeNP) against lipid peroxidation. The similar oxidative stability of meat across the broilers fed the Se supplemented diets, clearly indicated that CS-SeNP had significant antioxidant potential, comparable to that of the commonly-used SS or SeY sources. It can be stated that the elemental Se from CS-SeNP was not only absorbed; it was also biologically active. The Se in the CS-SeNP of the present study was in an elemental state $\left(\mathrm{Se}^{0}\right)$ as determined by the analyses. Elemental Se was thought to be biologically inert until Zhang., et al. [42] challenged this theory by proving that Se with zero valence state was very active. This was confirmed in a more recent study, where dietary elemental Se was found to be utilized as selenoprotein source [43].

Positive effects of dietary SeNP supplementation on the oxidative resistance of the organism have been reported in earlier studies with broilers [10,18-20] and fish [7]. The results of those works clearly indicate the association of supplemental SeNP with the elevation of the glutathione peroxidase activities in serum, kidney, liver, and muscle of the animals. However, in one study of Saleh and Ebeid [19], no effect of SeNP on muscle MDA was observed, most probably because MDA was not measured after iron-induced oxidation to determine the resistance of meat against lipid peroxidation. In general, a single measurement of MDA may not give reliable results in contrast to iron-induced oxidation, which correlates well with results from chill storage experiments and adequately indicates the relative oxidative stability and shelf-life of meat [38].

No major differences in the breast meat FA composition were

Citation: E Giamouri, et al. "Effects of Selenium Nanoparticles-loaded Chitosan Microspheres on Meat Selenium Content and Oxidative Stability in Broiler Chickens". Acta Scientific Veterinary Sciences 3.11 (2021): 27-38. 
detected between diets. Total polyunsaturated FA (PUFA), long chain (> 20 carbons) PUFA and long chain (> 20 carbons) n-3 PUFA, which are prone to oxidation, exhibited similar percentages in the broilers of all diets (Table 5).

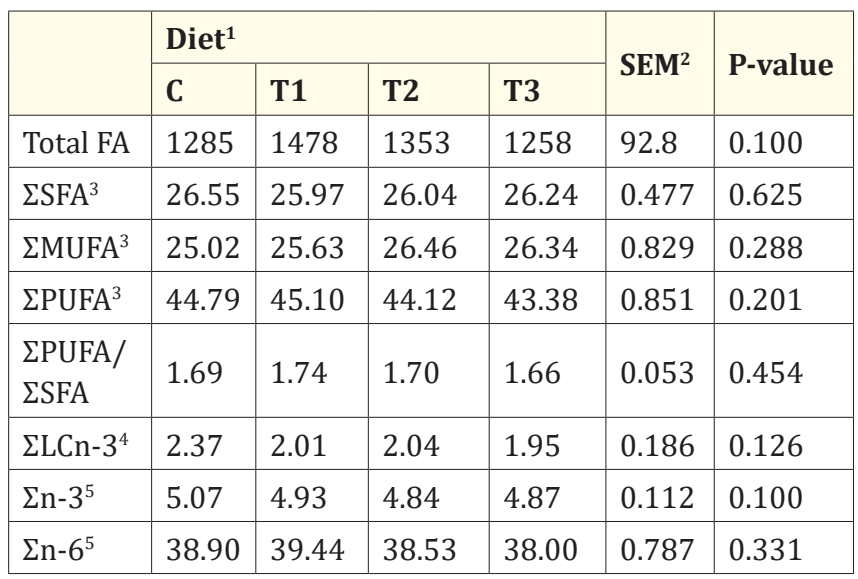

Table 5: Effects of diet on total fatty acids (mg/100 g wet tissue) and main fatty acid (FA) classes (\% of total FA) of breast in 42 day-old broilers ( $\mathrm{n}=10$ broilers/diet).

1. Se source: a) sodium selenite $(0.2 \mathrm{mg} / \mathrm{kg})$ and Se-yeast $(0.2 \mathrm{mg} /$ $\mathrm{kg}$ ) in T1 diet, b) Se-yeast ( $0.2 \mathrm{mg} / \mathrm{kg}$ ) and CS-SeNP (selenium nanoparticles-loaded chitosan microspheres, $0.2 \mathrm{mg} / \mathrm{kg}$ ) in T2 diet and c) CS-SeNP $(0.4 \mathrm{mg} / \mathrm{kg})$ in T3 diet; Se-yeast was in the form of Sel-Plex ${ }^{\circledR}$ (Alltech Inc., Nicholasville, KY, USA); no Se was added to the control (C) diet.

${ }^{2} \mathrm{SEM}=$ standard error of means.

3. $\Sigma S F A=$ sum of saturated fatty acids $(12: 0+14: 0+15: 0+16: 0+17: 0+$ $18: 0), \Sigma M U F A=$ sum of monounsaturated fatty acids $(14: 1+16: 1 \mathrm{n}-$ $7+17: 1+18: 1 n-9+18: 1 n-7+20: 1 n-9), \Sigma P U F A=$ sum of polyunsaturated fatty acids $(18: 2 n-6+18: 3 n-3+18: 3 n-6+20: 3 n-6+20: 3 n-$

$3+20: 4 n-6+20: 5 n-3+22: 4 n-6+22: 5 n-3+22: 6 n-3)$.

${ }^{4} \Sigma \mathrm{LCn}-3=$ sum of long carbon chain $(\geq 20 \mathrm{C}) \mathrm{n}-3$ fatty acids $(20: 3 n-$ $3+20: 5 n-3+22: 5 n-3+22: 6 n-3)$

5. $\Sigma \mathrm{n}-3=$ sum of $n-3$ fatty acids $(18: 3 n-3+20: 5 n-3+22: 5 n-$

$3+22: 6 n-3), 2 n-6=$ sum of n- 6 fatty acids $(18: 2 n-6+18: 3 n-$ $6+20: 3 n-6+20: 4 n-6+22: 4 n-6)$.

In general, the effects of Se from CS-SeNP on the FA composition of meat from livestock has not been investigated so far; there- fore, we do not have any literature for comparison purposes. In a previous study with broilers, organic Se was found to promote an increase in the long chain PUFA and n-3 PUFA, in addition to enhanced oxidative stability [44]. Similarly, it was reported that dietary supplementation with organic Se in rabbits improved muscle FA composition in comparison with an unsupplemented diet [38]. No such effect was observed herein. It has been postulated that Se can produce changes in PUFA metabolism analogous to those elicited by vitamin E [37]. Indeed, in a study investigating the effect of Se deficiency on FA composition of liver, rats fed Sedeficient diets, compared to rats fed Se-adequate diets, exhibited reduced levels of palmitic acid and long chain PUFA like DHA, and increased levels of n-6 FA such as linoleic acid [45]. Therefore, the above mentioned studies in broilers and rabbits indicate that Se could be implicated in the peroxisomal $\beta$-oxidation, which has been reported to increase with the dietary Se content. However, none of these studies $[38,44]$ used Se-deficient diets, and so was the case in the present work; hence, it appears that the effects of Se on meat FA composition are much more complicated and do not necessarily depend on whether diets are Se-deficient or not. Nonetheless, the changes in meat FA composition induced by Se supplementation were accompanied by increased oxidative stability, indicating that Se may have played both an in vivo metabolic, and a post-mortem protective role $[38,44]$. On the other hand, our work was able to detect only the post-mortem protective role of dietary Se (enhanced oxidative stability of breast meat), but no changes in the breast FA composition were found. This was obvious for all the Se sources used herein. Further research is necessary to elucidate under what conditions the role of dietary Se on FA metabolism is exerted.

\section{Conclusion}

Breast Se content readily increased by the dietary supplementation with $0.4 \mathrm{mg}$ Se from CS-SeNP, resulting in Se enriched meat similarly to the commonly used Se sources. The dietary CS-SeNP also improved breast oxidative stability in a manner comparable to the commonly used Se sources. The present results indicate that CS-SeNP can be a potential source of bioavailable Se with an important protective role against meat oxidation and merits further investigation in broiler feeding.

\section{Acknowledgements}

This Research Project is co-financed by Greece and the European Union (European Social Fund) through the Operational Program 
«Human Resources Development, Education and Lifelong Learning 2014-2020» and the Program encoded EDBM103, titled "Support for researchers with an emphasis on young researchers-cycle B '”, in the context of the project "Innovative nanomaterials in animal feeding: evaluation of selenium nanoparticles-loaded chitosan microspheres on meat quality" (MIS 5048474). We are grateful to Bouzas, E. and Danezis, G. for their help during selenium analysis.

\section{Conflict of Interest}

No financial interest or any conflict of interest exists.

\section{Acknowledgment}

Dr. L. Sygellou (FORTH/ICE-HT) and Dr. V. Dracopoulos (FORTH/ ICE-HT) are thanked for XPS and SEM measurements/analysis, respectively.

\section{Bibliography}

1. Ringuet MT., et al. "Analysis of bioavailability and induction of glutathione peroxidase by dietary nanoelemental, organic and inorganic selenium". Nutrients 13 (2021): 1073.

2. Korzeniowska M., et al. "Effect of dietary selenium on protein and lipid oxidation and the antioxidative potential of selected chicken culinary parts during frozen storage". Journal of Chemistry 2018 (2018): 1-12.

3. Wang CL., et al. "Effects of selenium source and level on growth performance, antioxidative ability and meat quality of broilers". Journal of Integrative Agriculture 20.1 (2021): 227-235.

4. Zhao L., et al. "A novel organic selenium compound exerts unique regulation of selenium speciation, selenogenome, and selenoproteins in broiler chicks". Journal of Nutrition 147.5 (2017): 789-797.

5. Khurana A., et al. "Therapeutic applications of selenium nanoparticles". Biomedicine and Pharmacotherapy 111.1 (2019): 802-812.

6. Rayman MP. "Selenium intake, status, and health: a complex relationship". Hormones 19 (2020): 9-14.

7. Araujo JM., et al. "Delivery of selenium using chitosan nanoparticles: synthesis, characterization, and antioxidant and growth effects in Nile tilapia (Oreochromis niloticus)". Plos One 16.5 (2021): e0251786.
8. EU. "Commission Regulation (EC) No 427/2013 of 8 May 2013 concerning the authorisation of selenomethionine produced by Saccharomyces cerevisiae NCYC R646 as a feed additive for all Animal species and amending Regulations (EC) No 1750/2006, (EC) No 634/2007 and (EC) No 900/2009 as regards the maximum supplementation with selenised Yeast". Official Journal of the European Union, L 127 (2013): 20-22.

9. Payne RL and Southern LL. "Changes in glutathione peroxidase and tissue selenium concentrations of broilers after consuming a diet adequate in selenium". Poultry Science 84.8 (2005): 1268-1276.

10. Cai SJ., et al. "Effects of nano - selenium on performance, meat quality, immune function, oxidation resistance, and tissue selenium content in broilers". Poultry Science 91.10 (2012): 2532-39.

11. Samak DH., et al. "Developmental toxicity of carbon nanoparticles during embryogenesis in chicken". Environmental Science and Pollution Research International 27.16 (2018): 1905819072.

12. Shahnawaz K., et al. "Impacts of selenium and vitamin E supplementation on mRNA of heat shock proteins, selenoproteins and antioxidants in broilers exposed to high temperature". AMB Express 8.1 (2018): 112.

13. Abdul H., et al. "Green synthesis and characterization of zinc oxide nanoparticles from Ocimum basilicum L. var purpurascens Benth.-lamiaceae leaf extract". Materials Letters 131 (2014): 16-18.

14. Li H., et al. "Synthesis and cytotoxicity of selenium nanoparticles stabilized by $\alpha$-D-glucan from Castanea mollissima Blume". International Journal of Biological Macromolecules 129 (2019): 818-826.

15. Hosnedlova B., et al. "Nano-selenium and its nanomedicine applications: a critical review". International Journal of Nanomedicine 13 (2018): 2107-2128.

16. Zhang JS., et al. "Elemental selenium at nano size (nano - se) as a potential chemopreventive agent with reduced risk of selenium toxicity: comparison with se - methylselenocysteine in mice". Toxicology Science 101.1 (2008): 22-31. 
17. Wang H., et al. "Elemental selenium at nano size possesses lower toxicity without compromising the fundamental effect on selenoenzymes: comparison with selenomethionine in mice". Free Radical Biology and Medicine 42.10 (2007): 15241533.

18. Zhou X and Wang Y. "Influence of dietary nano elemental selenium on growth performance, tissue selenium distribution, meat quality and glutathione peroxidase activity in Guangxi Yellow Chicken". Poultry Science 90.3 (2011): 680-686.

19. Saleh AA and Ebeid TA. "Feeding sodium selenite and nanoselenium stimulates growth and oxidation resistance in broilers". South African Journal of Animal Science 49.1 (2019): 176184.

20. Aparna N and Karunakaran R. "Effect of selenium nanoparticles supplementation on oxidation resistance of broiler chicken". Indian Journal of Science and Technology 9.1 (2016): 1-5.

21. Li B., et al. "Biogenic selenium and its hepatoprotective activity”. Scientific Reports 7 (2017): 15627.

22. Bai K., et al. "Preparation and antioxidant properties of selenium nanoparticles-loaded chitosan microspheres". International Journal of Nanomedicine 12 (2017): 4527-4539.

23. Ren L., et al. "Preparation and growth-promoting effect of selenium nanoparticles capped by polysaccharide-protein complexes on tilapia". Journal of the Science of Food and Agriculture 101.2 (2021): 476-485.

24. Filipović N., et al. "Comparative study of the antimicrobial activity of selenium nanoparticles with different surface chemistry and structure". Frontiers in Bioengineering and Biotechnology 8 (2021): 1-16.

25. Bai K., et al. "Selenium nanoparticles-loaded chitosan/citrate complex and its protection against oxidative stress in d-galactose-induced aging mice". Journal of Nanobiotechnology 15.92 (2017): 1-14.

26. Pappas AC., et al. "Effects of supplementing broiler breeder diets with organoselenium compounds and polyunsaturated fatty acids on hatchability". Poultry Science 85.9 (2006): 15841593.
27. O'Fallon JV., et al. "A direct method for fatty acid methylester synthesis: application to wet meat tissues, oils, and feedstuffs". Journal Animal Science 85.6 (2007): 1511-1521.

28. Gatellier P., et al. "Effect of diet finishing mode (pasture or mixed diet) on antioxidant status of charolais bovine meat". Meat Science 67.3 (2004): 385-394.

29. Terevinto A., et al. "Oxidative status, in vitro iron-induced lipid oxidation and superoxide dismutase, catalase and glutathione peroxidase activities in rhea meat". Meat Science 84.4 (2010): 706-710.

30. Sreekumar S., et al. "Parameters influencing the size of chitosan-TPP nano- and microparticles". Scientific Reports 8.1 (2018): 4695.

31. Abid S., et al. "Synthesis and characterization of glycol chitosan coated selenium nanoparticles acts synergistically to alleviate oxidative stress and increase ginsenoside content in Panax ginseng". Carbohydrate Polymers 267 (2021): 118195.

32. Zhang C., et al. "Synthesis, characterization, and controlled release of selenium nanoparticles stabilized by chitosan of different molecular weights". Carbohydrate Polymers 134 (2015): 158-166.

33. Filipović, N., et al. "Poly (e-caprolactone) microspheres for prolonged release of selenium nanoparticles". Materials Science Engineering 96 (2019): 776-789.

34. Chung S., et al. "Green synthesized BSA-coated selenium nanoparticles inhibit bacterial growth while promoting mammalian cell growth". International Journal of Nanomedicine 15 (2020): 115-124.

35. Dlouhá G., et al. "Effect of dietary selenium sources on growth performance, breast muscle selenium, glutathione peroxidase activity and oxidative stability in broilers". Czech Journal of Animal Science 53.6 (2008): 265-269.

36. Zhang Y., et al. "The effect of dietary selenium levels on growth performance, antioxidant capacity and glutathione peroxidase 1 (GSHPx1) mRNA expression in growing meat rabbits". Animal Feed Science and Technology 169.3-4 (2011): 259-264. 
37. Ebeid TA., et al. "Fortification of rabbit diets with vitamin E or selenium affects growth performance, lipid peroxidation, oxidative status and immune response in growing rabbit". Livestock Science 155.2-3 (2013): 323-331.

38. Papadomichelakis G., et al. "Effects of increasing dietary organic selenium levels on meat fatty acid composition and oxidative stability in growing rabbits". Meat Science 131 (2017): 132-138.

39. $\mathrm{Hu} \mathrm{CH}$. , et al. "Comparative effects of nano elemental selenium and sodium selenite on selenium retention in broiler chickens". Animal Feed Science and Technology 177.3-4 (2012): 204-210.

40. Zhang J., et al. "Development, physicochemical characterization and cytotoxicity of selenium nanoparticles stabilized by beta-lactoglobulin". International Journal of Biological Macromolecules 107 (2018): 1406-1413.

41. Bai K., et al. "Selenium-nanoparticles-loaded chitosan/chitooligosaccharide microparticles and their antioxidant potential: a chemical and in vivo investigation". Pharmaceutics 12.1 (2020): 43 .

42. Zhang JS., et al. "Biological effects of a nano red elemental selenium”. Biofactors 15.1 (2001): 27-38.

43. Hadrup N., et al. "Effects of 14-day oral low dose selenium nanoparticles and selenite in rat-As determined by metabolite pattern determination". Peer Journal 4 (2016): e2601.

44. Pappas AC., et al. "Supranutritional selenium level affects fatty acid composition and oxidative stability of chicken breast muscle tissue". Journal of Animal Physiology and Animal Nutrition 96.3 (2012): 385-394.

45. Schäfer K., et al. "Effects of selenium deficiency on fatty acid metabolism in rats fed fish oil-enriched diets". Journal of Trace Elements in Medicine and Biology 18.1 (2004): 89-97.

Volume 3 Issue 11 November 2021

(C) All rights are reserved by E Giamouri., et al. 